\title{
Input-Output Decoupling with Stability for Hamiltonian Systems*
}

\author{
H. J. C. Huijberts $\dagger$ and A. J. van der Schaft $\dagger$
}

\begin{abstract}
The input-output decoupling problem with stability for Hamiltonian systems is treated using decoupling feedbacks, all of which make the system maximally unobservable. Using the fact that the dynamics of the maximal unobservable subsystem are again Hamiltonian, an easily checked condition for inputoutput decoupling with (critical) stability is deduced.
\end{abstract}

Key words. Decoupled systems, Critical stability, Unobservable dynamics, Constrained Hamiltonian systems.

\section{Introduction}

While the solution of the input-output decoupling problem for nonlinear control systems is well established (see [I] and [NS] for references), relatively little attention has been paid in the literature to the problem of finding a decoupling feedback which also achieves internal stability of the closed-loop nonlinear system (see, however, [IG], received during preparation of this paper).

It is well known that the nonlinear (scalar) input-(scalar) output decoupling problem is solvable if and only if the decoupling matrix, a nonlinear generalization of the Falb-Wolovich matrix from linear theory, has full rank everywhere. Furthermore, with the aid of this decoupling matrix a large class of decoupling feedbacks, which all have the property that they decouple the system into $m$ linear inputoutput systems (with $m$ being the number of inputs and outputs), together with an unobservable nonlinear system (which actually is the maximal subsystem that can be made unobservable; see [I] for references), can be constructed.

In this paper we táckle the problem of input-output decoupling with stability for Hamiltonian systems, using the same easily computable class of decoupling feedbacks as indicated above. In [S2] and [S3] it was shown that the remaining unobservable nonlinear system in this case is a Hamiltonian subsystem of the original system; in fact, its dynamics are governed by a Hamiltonian function $\bar{H}$, which is simply obtained by restricting the original Hamiltonian function $H$ (energy) to a symplectic submanifold which forms the state space for this unobservable non-

\footnotetext{
* Date received: August 13, 1987. Date revised: June 7, 1988.

$\dagger$ Department of Applied Mathematics, University of Twente, P.O. Box 217, 7500 AE Enschede, The Netherlands.
} 
linear system. It follows that these unobservable dynamics are at most critically, but never asymptotically, stable. The main result of this paper states that if the Hamiltonian $\bar{H}$ has an isolated (local) minimum at the equilibrium point-a condition which can be easily verified, and which forms a sufficient (and in many cases generically also a necessary) condition for stability of the unobservable dynamicsthen by making the linear decoupled input-output systems separately asymptotically stable by static feedback the whole closed-loop system becomes locally stable. This is proved by an application of center manifold theory, as introduced in nonlinear control theory in [A] (see also [M2]).

As an important preliminary result we derive a property of the canonical form for general (not necessarily Hamiltonian) input-output decoupled systems, which is of independent interest: the coordinates for the maximal unobiservable system can be chosen in such a way that this subsystem is not directly influenced by the inputs if and only if the distribution of input vector fields is involutive.

In this paper asymptotic stability of the input-output decoupled Hamiltonian system is only achieved if the maximal unobservable system is zero-dimensional. However, it is easy to give examples of Hamiltonian systems with nontrivial maximal unobservable dynamics which can be made asymptotically stable and inputoutput decoupled at the same time. This is because we have restricted attention to a large, but proper subset of all decoupling feedbacks. In [HG] the set of all decoupling feedbacks was characterized (generalizing the linear results of [G]), while in [IG] (see also [NS]) the dynamics that are left invariant by all decoupling feedbacks are determined. These dynamics are contained in the maximal unobservable dynamics. This notion of fixed dynamics deserves a closer study in the Hamiltonian case, and is expected to lead to an extension of the results obtained in this paper. This remains for further research.

After having submitted the first version of this paper we received a preprint of [BI1], dealing with stabilizability of general (non-Hamiltonian) systems, where methods related to the ones used in this paper are employed. For results on the general feedback stabilization problem we refer to $[\mathrm{B}]$ and the references therein and to [BB, BI2], and [T].

\section{Hamiltonian Systems}

We briefly review the definition of a Hamiltonian system, see, e.g., [S1] and [CS]. Let $M$ be a $2 n$-dimensional connected manifold with symplectic form $\omega$. By Darboux's theorem there exist local coordinates $(q, p)=\left(q_{1}, \ldots, q_{n}, p_{1}, \ldots, p_{n}\right)$ such that $\omega=\sum_{i=1}^{n} d p_{i} \wedge d q_{i}$. Such coordinates are called canonical. Given a function $F: M \rightarrow \mathbb{R}$ we define the Hamiltonian vector field $X_{F}$ on $M$ by $\omega\left(X_{F}, \cdot\right)=-d F$. In canonical coordinates [AM]

$$
X_{F}=\sum_{i=1}^{n}\left(\frac{\partial F}{\partial p_{i}} \frac{\partial}{\partial q_{i}}-\frac{\partial F}{\partial q_{i}} \frac{\partial}{\partial p_{i}}\right) .
$$

Given another function $G: M \rightarrow \mathbb{R}$ we define the Poisson bracket $\{F, G\}=X_{F}(G)=$ $\omega\left(X_{F}, X_{G}\right)$. In canonical coordinates

$$
\{F, G\}=\sum_{i=1}^{n}\left(\frac{\partial F}{\partial p_{i}} \frac{\partial G}{\partial q_{i}}-\frac{\partial F}{\partial q_{i}} \frac{\partial G}{\partial p_{i}}\right) .
$$


A (coordinate) transformation $\varphi: M \rightarrow M$ is canonical if $\varphi$ preserves the Poisson bracket, i.e.,

$$
\{F \circ \varphi, G \circ \varphi\}=\{F, G\} \circ \varphi \quad \text { for all } F, G .
$$

An (affine) Hamiltonian system on $M$ with internal energy $H$ is now defined as

$$
\dot{x}=X_{H}(x)-\sum_{j=1}^{m} u_{j} X_{C_{j}}(x), \quad y_{j}=C_{j}(x),
$$

with $x$ the local (possibly canonical) coordinates, $u=\left(u_{1}, \ldots, u_{m}\right)$ the inputs, $y=$ $\left(y_{1}, \ldots, y_{m}\right)$ the outputs, and $C=\left(C_{1}, \ldots, C_{m}\right)$ the output mapping. This constitutes a direct generalization of the classical Euler-Lagrange equations with external forces $u_{i}$

$$
\begin{aligned}
\frac{d}{d t}\left(\frac{\partial L}{\partial \dot{q}_{i}}\right)-\frac{\partial L}{\partial q_{i}} & = \begin{cases}u_{i}, & i=1, \ldots, m, \\
0, & i=m+1, \ldots, n,\end{cases} \\
y_{j} & =q_{j}, \quad j=1, \ldots, m,
\end{aligned}
$$

or in Hamiltonian form (with $p_{i}=\partial L / \partial \dot{q}_{i}$ the momenta and $H(q, p)=$ $\sum_{i=1}^{n} \dot{q}_{i}\left(\partial L / \partial \dot{q}_{i}\right)-L(q, \dot{q})$ the internal energy)

$$
\begin{array}{ll}
\dot{q}_{i}=\frac{\partial H}{\partial p_{i}}, & i=1, \ldots, n, \\
\dot{p}_{i}=-\frac{\partial H}{\partial q_{i}}+u_{i}, & i=1, \ldots, m, \\
\dot{p}_{i}=-\frac{\partial H}{\partial q_{i}}, & i=m+1, \ldots, n, \\
y_{j}=q_{j}, & j=1, \ldots, m .
\end{array}
$$

In fact, if we take $C_{j}(q, p)=q_{j}$ in (2.4) we obtain (2.6), and conversely if we allow for canonical coordinate transformations in (2.6), then (2.6) takes the form (2.4).

A particular subclass of Hamiltonian systems often encountered in applications, e.g., involving mechanical systems, is given below:

Definition 2.1. Let $M$ be of the form $T^{*} Q$ with $Q$ the configuration manifold with coordinates $\left(q_{1}, \ldots, q_{n}\right)$. Let $H(q, p)=K(q, p)+V(q)$, where $K(q, p)=$ $\frac{1}{2} \sum_{i, j=1}^{n} g^{i j}(q) p_{i} p_{j}$ and the matrix $\left(g^{i j}(q)\right)$ is positive definite, and let $C_{1}, \ldots$, $C_{m}: Q \rightarrow \mathbb{R}$. Then the Hamiltonian system (2.4) is called simple. The term $K(q, p)$ is called the kinetic energy and the term $V(q)$ is called the potential energy of the system.

\section{The Canonical Form for Decoupled Systems}

We consider a general nonlinear system on a smooth manifold $M$, given in local coordinates by

$$
\begin{aligned}
& \dot{x}=f(x)+\sum_{i=1}^{m} u_{i} g_{i}(x), \\
& y_{j}=h_{j}(x), \quad j=1, \ldots, m,
\end{aligned}
$$


where $f, g_{1}, \ldots, g_{m}$ are smooth vector fields on $M$ and $h_{1}, \ldots, h_{m}$ are smooth real-valued functions on $M$.

For (3.1) we define characteristic numbers $\rho_{1}, \ldots, \rho_{m}$ by

$$
\rho_{i}(x)=\min _{k \in \mathbb{N}}\left(\text { for some } j \in \mathrm{m}: L_{g_{j}} L_{j}^{k} h_{i}(x) \neq 0\right) \text {. }
$$

(Here and in the following $m$ denotes the set $\{1, \ldots, m\}$ for $m \in \mathbb{N}$.) Assume that $\rho_{i}(x)$ does not depend on $x$, i.e., $\rho_{i}(x)=\rho_{i}$ and $\rho_{i}<\infty(i \in \mathbf{m})$. Then we define the decoupling matrix $A(x)$ for (3.1) as the matrix with elements

$$
a_{i j}(x)=L_{g_{j}} L_{f}^{\rho_{i}} h_{i}(x) \quad(i, j \in \mathbf{m}) .
$$

It is well known (see [I]) that the input-output decoupling problem is solvable for all $x \in M$ if and only if the decoupling matrix $A(x)$ has fulf rank for all $x \in M$. Moreover, if $A(x)$ has full rank for all $x \in M$, the functions $h_{1}(x), \ldots, L_{f}^{\rho_{1}} h_{1}(x), \ldots$, $h_{m}(x), \ldots, L_{f}^{\rho_{m}} h_{m}(x)$ are independent for all $x \in M$. Hence, under this assumption we can choose coordinates $\left(\xi_{1}, \ldots, \xi_{m+1}\right)$ for $M$ in the following way:

$$
\begin{aligned}
\xi_{i} & =\left[\begin{array}{c}
z_{i 0} \\
\vdots \\
z_{i \rho_{i}}
\end{array}\right]=\left[\begin{array}{c}
h_{i}(x) \\
\vdots \\
L_{f}^{\rho_{i}} h_{i}(x)
\end{array}\right] \quad(i \in \mathbf{m}), \\
\xi_{m+1} & =\left[\begin{array}{c}
z_{m+1,1} \\
\vdots \\
z_{m+1, d}
\end{array}\right] \quad \text { where } \quad d=n-\sum_{i=1}^{m}\left(\rho_{i}+1\right) .
\end{aligned}
$$

A large class of decoupling feedbacks $u_{i}=\alpha_{i}(x)+\sum_{j=1}^{m} \beta_{i j}(x) v_{j}$ (with the matrix $\left(\beta_{i j}(x)\right)_{i, j \in \mathrm{m}}$ invertible for all $x \in M$ and $v_{1}, \ldots, v_{m}$ the new inputs) is given by the solutions of (see [I])

$$
\left.\begin{array}{rl}
A_{i}(x) \alpha(x)+b_{i}(x) & =\gamma_{i}(x) \\
A_{i}(x) \beta(x) & =\delta_{i}(x)
\end{array}\right\} \quad(i \in \mathbf{m}),
$$

where $A_{i}(x)$ is the $i$ th row of the decoupling matrix $A(x), b_{i}(x)=L_{f}^{p_{i}+1} h_{i}(x)$, $\gamma_{i}(x)$ is a function whose differential belongs to $\operatorname{span}\left\{d L_{f}^{k} h_{i} ; 0 \leq k \leq \rho_{i}\right\}$, and $\delta_{i}(x)$ is a row vector with $i$ th element a function whose differential belongs to $\operatorname{span}\left\{d L_{f}^{k} h_{i} ; 0 \leq k \leq \rho_{i}\right\}$ and other elements equal zero.

In particular we can take $\gamma_{i}(x)$ to be a linear function of $\xi_{i}$, say $\gamma_{i}(x)=\sum_{j=0}^{p_{i}} \varepsilon_{i j} z_{i j}$, and the nonzero element of $\delta_{t}(x)$ to be constant, say $\delta_{i i}(x)=\sigma_{i} \in \mathbb{R}$. The class of decoupling feedbacks of this type is denoted by $\mathscr{L}$. In the coordinates (3.4) the decoupled system has the following structure after application of a decoupling feedback from the class $\mathscr{L}$ (see [I]):

$$
\begin{aligned}
\dot{\xi}_{i} & =A_{i} \xi_{i}+B_{i} v_{i} \quad(i \in \mathbf{m}), \\
\dot{\xi}_{m+1} & =\bar{f}\left(\xi_{1}, \ldots, \xi_{m+1}\right)+\sum_{j=1}^{m} \bar{g}_{j}\left(\xi_{1}, \ldots, \xi_{m+1}\right) v_{j},
\end{aligned}
$$

where $A_{i}$ is the constant $\left(\rho_{i}+1, \rho_{i}+1\right)$ matrix

$$
\left[\begin{array}{ccccc}
0 & 1 & 0 & \cdots & 0 \\
0 & 0 & 1 & \cdots & 0 \\
\vdots & & & \ddots & \vdots \\
\vdots & & & & 1 \\
\varepsilon_{i 0} & \varepsilon_{i 1} & \cdots & \cdots & \varepsilon_{i \rho_{i}}
\end{array}\right]
$$


$B_{i}$ is the constant $\left(\rho_{i}+1\right)$ vector

$$
\left[\begin{array}{c}
0 \\
\vdots \\
0 \\
\sigma_{i}
\end{array}\right],
$$

and $\bar{f}, \bar{g}_{j}(j \in \mathbf{m})$ depend upon the choice of the coordinates $\xi_{m+1}$.

We see from (3.4) that the outputs do not depend on $\xi_{m+1}$. Note that, in fact, $\xi_{m+1}$ are coordinates for the maximal controlled invariant distribution [I] contained in $\bigcap_{i=1}^{m} \operatorname{Ker} d h_{i}$, which we call $D^{*}$ in the following.

It is natural to question whether the coordinates $\xi_{m+1}$ for $D^{*}$ can be chosen in such a way that the dynamics of $\xi_{m+1}$ are not directly influenced by the inputs, i.e., $\bar{g}_{j}\left(\xi_{1}, \ldots, \xi_{m+1}\right)=0(j \in \mathbf{m})$ in (3.6). (For $m=1$ this question was answered affirmatively in [M1].) Because $\beta(x)$ is assumed to have full rank this is the case if and only if $\xi_{m+1}$ can be chosen in such a way that in the original system (before feedback) the dynamics of $\xi_{m+1}=\left(z_{m+1,1}, \ldots, z_{m+1, d}\right)$ are not influenced by the old inputs, i.e.,

$$
L_{g_{j}} z_{m+1, k}=0 \quad \text { for all } j \in \mathbf{m}, k \in \mathbf{d},
$$

or, equivalently, $z_{m+1, k} \in G^{\perp}$, for all $k \in \mathrm{d}$, where $G$ is the distribution spanned by the input vector fields $g_{1}, \ldots, g_{m}$ and $G^{\perp}$ is the annihilating codistribution of $G$. (From (3.3) and the assumption rank $A=m$ it immediately follows that the dimension of $G$ is constant, $m$.) Since $\xi_{m+1}$ has to be such that $\left(\xi_{1}, \ldots, \xi_{m+1}\right)$ form a coordinate system, the following conditions on $\xi_{m+1}$ also have to be satisfied:

$$
\begin{gathered}
d z_{m+1, k} \notin F:=\operatorname{span}\left\{d L_{f}^{k} h_{i} ; 0 \leq k \leq \rho_{i}, i \in \mathbf{m}\right\} \quad(k \in \mathbf{d}), \\
\operatorname{dim} \operatorname{span}\left\{d z_{m+1,1}, \ldots, d z_{m+1, d}\right\}=d .
\end{gathered}
$$

Before giving the final result, we first prove two technical lemmas.

Lemma 3.1. If $A(x)$ has rank $m$ for all $x \in M$, then $G \cap F^{\perp}=\{0\}$.

Proof. Assume $\alpha_{1} g_{1}+\cdots+\alpha_{m} g_{m} \in F^{\perp}$ for some functions $\alpha_{1}, \ldots, \alpha_{m}$. Then, for $i \in \mathbf{m}$,

$$
\left\langle d L_{f}^{\rho_{i}} h_{i}, \alpha_{1} g_{1}+\cdots+\alpha_{m} g_{m}\right\rangle=\sum_{j=1}^{m} \alpha_{j} L_{g_{j}} L_{f}^{\rho_{i}} h_{i}=0 .
$$

Hence $\sum_{j=1}^{m} \alpha_{j} A^{j} \stackrel{?}{=} 0$, where $A^{j}$ is the $j$ th column of $A$. By nonsingularity of $A$ this implies $\alpha_{1}=\cdots=\alpha_{m}=0$.

Lemma 3.2. If $A(x)$ has rank $m$ for all $x \in M$, then

$$
G^{\perp} \cap F=\operatorname{span}\left\{d L_{f}^{k} h_{i} ; 0 \leq k \leq \rho_{i}-1, i \in \mathbf{m}\right\} .
$$

Proof. From the fact that $L_{g_{j}} L_{f}^{k} h_{i}=0$ for all $j \in \mathrm{m}, 0 \leq k \leq \rho_{i}-1$, it follows that $\operatorname{span}\left\{d L_{f}^{k} h_{i} ; 0 \leq k \leq \rho_{i}-1, i \in \mathbf{m}\right\} \subset G^{\perp} \cap F$. Moreover, from the definition of the characteristic numbers it follows that $\left\langle d L_{f}^{\rho_{i}} h_{i}, g_{j}\right\rangle=L_{g_{j}} L_{f}^{\rho_{i}} h_{i} \neq 0$ for at least one $j \in \mathbf{m}$, so $d L_{f}^{\rho_{i}} h_{i} \notin G^{\perp}$. Hence $G^{\perp} \cap F=\operatorname{span}\left\{d L_{f}^{k} h_{i} ; 0 \leq k \leq \rho_{i}-1, i \in \mathbf{m}\right\}$, as claimed. 
Now we come to the final result (see also [MBE]).

Theorem 3.1. Assume that rank $A(x)=m$ for all $x \in M$. Then there are smooth functions $z_{1}, \ldots, z_{d}: M \rightarrow \mathbb{R}$, with $d=n-\sum_{i=1}^{m}\left(\rho_{i}+1\right)$, that satisfy (3.7)-(3.9) if and only if $G$ is involutive.

Proof. (Necessity) Assume that $z_{1}, \ldots, z_{d}$ satisfying (3.7)-(3.9) exist. From Lemma 3.1 we have $G \cap F^{\perp}=\{0\}$ or, equivalently, $G^{\perp}+F=T^{*} M$. So $\operatorname{dim}\left(G^{\perp} \backslash F\right)=$ $n-\operatorname{dim}(F)=d$. From this fact and (3.7)-(3.9) it follows that $G^{\perp} \backslash F=$ $\operatorname{span}\left\{d z_{1}, \ldots, d z_{d}\right\}$. Moreover, by Lemma $3.2 G^{\perp}=\operatorname{span}\left\{\left(d L_{f}^{k} h_{i} ; 0 \leq k \leq \rho_{i}-1\right.\right.$, $\left.i \in \mathbf{m}), d z_{1}, \ldots, d z_{d}\right\}$. Hence $G^{\perp}$ is spanned by exact one-forms and this implies that $G$ is involutive.

(Sufficiency) Involutivity of $G$ implies that $G^{\perp}$ can be spanned by $n-m$ exact one-forms. The functions that define the exact one-forms spanning $G^{\perp} \backslash F$ satisfy (3.7)-(3.9). So there are functions $z_{1}, \ldots, z_{d}$ satisfying (3.7)-(3.9).

Remarks. (1) For linear systems, $G$ is always involutive, so in this case we can always choose coordinates $\xi_{m+1}$ for $D^{*}$ (which in this case is a linear subspace) such that the dynamics of $\xi_{m+1}$ are not directly influenced by the inputs. (This also follows from the Morse canonical form.)

(2) We see that if $G$ is involutive, then the decoupled system has the structure shown in Fig. 1 in coordinates $\xi_{1}, \ldots, \xi_{m+1}$ as above.

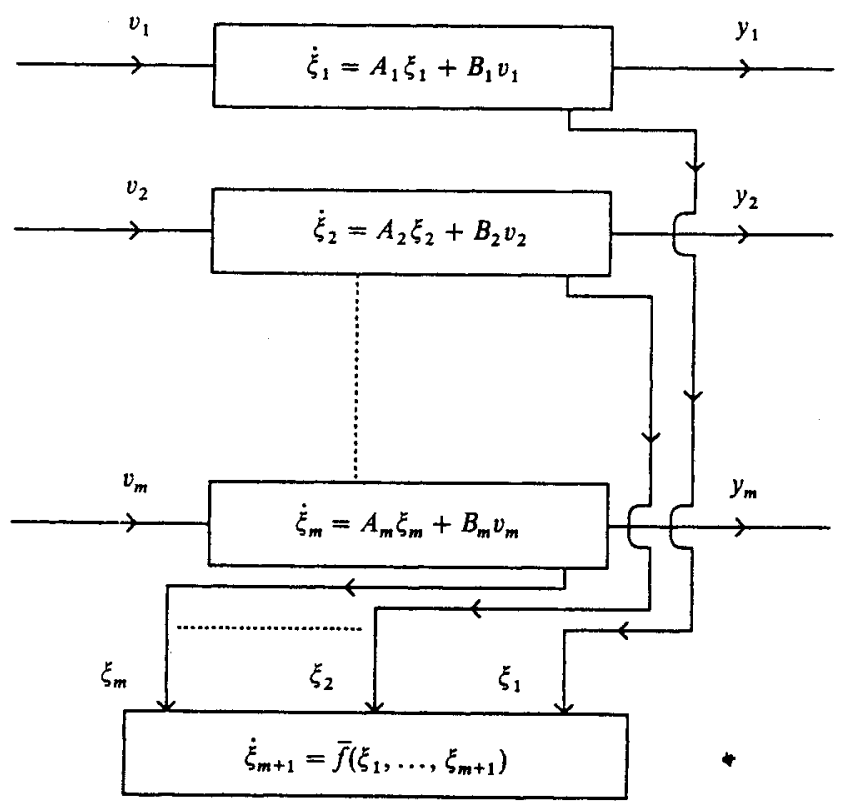

Fig. 1. Structure of decoupled system when $G$ is involutive. 


\section{The Input-Output Decoupling Problem with Stability for Hamiltonian Systems}

Let us now consider a Hamiltonian system on a symplectic manifold $(M, \omega)$ (see Section 2).

$$
\begin{aligned}
& \dot{x}=X_{H}(x)-\sum_{j=1}^{m} u_{j} X_{C_{j}}(x), \\
& y_{j}=C_{j}(x), \quad j \in \mathbf{m},
\end{aligned}
$$

where $H, C_{1}, \ldots, C_{m} \in C^{\infty}(M)$. We assume throughout that the input-output decoupling problem for (4.1) is solvable, i.e., that the decoupling matrix $A(x)$ associated with (4.1) (see Section 3) is nonsingular everywhere. Notice that by the definition of the Poisson bracket $(2.2)$ the $(i, j)$ th element of $A(x)$ can be rewritten as (see [S2] and $[\mathrm{S} 3])$

$$
a_{i j}(x)=L_{X_{c_{j}}} L_{X_{H}}^{\rho_{i}} C_{i}(x)=\left\{C_{j}, \operatorname{ad}_{H}^{\rho_{i}} C_{i}\right\}(x),
$$

where we define inductively $\operatorname{ad}_{H}^{0} C_{i}=C_{i}, \operatorname{ad}_{H}^{k} C_{i}=\left\{H, \operatorname{ad}_{H}^{k-1} C_{i}\right\}, k \geq 1$, and where $\rho_{i}$ is the smallest integer such that there exists $j \in \mathbf{m}$ for which $\left\{C_{j}, \operatorname{ad}_{H}^{\rho} C_{i}\right\}(x) \neq 0$. It follows that in order to obtain the decoupling matrix $A(x)$ we do not have to compute $X_{H}$ and $X_{c_{j}}, j \in \mathbf{m}$, explicitly; knowledge of the energy $H$ and the observation functions $C_{j}, j \in \mathrm{m}$, suffices.

We also assume throughout that the distribution $G=\operatorname{span}\left\{X_{C_{1}}, \ldots, X_{C_{m}}\right\}$ is involutive. For Hamiltonian systems this assumption is not very restrictive, as is seen from the following lemma:

Lemma 4.1. Consider the Hamiltonian system (4.1). Then $G$ is involutive if $\rho_{i}>0$ $(i \in \mathbf{m})$.

Proof. Assume that $\rho_{i}>0(i \in \mathbf{m})$. Then, for all $i, j \in \mathbf{m},\left[X_{C_{i}}, X_{C_{j}}\right]=X_{\left\{c_{i}, C_{j}\right\}}=$ $X_{0}=0 \in G$. Hence $G$ is involutive.

Remark. For simple Hamiltonian systems all characteristic numbers equal one, and hence for simple Hamiltonian systems $G$ is always involutive.

From Section 3 we now know that we can apply a decoupling feedback from the class $\mathscr{L}$ to (4.1), so that the decoupled system consists of $m$ linear singleinput single-output systems $\Sigma_{i}(i \in \mathbf{m})$ together with a nonlinear system $\tilde{\Sigma}$, whose dynamics are not directly influenced by the inputs. Furthermore, we know from [S2] and [S3] that in the Hamiltonian case the state space of this nonlinear system $\tilde{\Sigma}$, denoted by $N^{*}$ (in fact, $N^{*}$ is the maximal controlled invariant submanifold contained in $\bigcap_{i=1}^{m} \operatorname{Ker} d C_{i}$ ), is a symplectic submanifold of $M$. Hence coordinates $\left(\xi_{i}(i \in \mathbf{m}), \tilde{q}, \tilde{p}\right)$ of $M$ can be chosen, with $\xi_{i}$ as in Section 3 and with $(\tilde{q}, \tilde{p})$ partial canonical coordinates, in which the decoupled system has the 
form

$$
\begin{aligned}
& \Sigma_{i}: \quad \dot{\xi}_{i}=A_{i} \xi_{i}+B_{i} v_{i} \quad(i \in \mathrm{m}), \\
& \tilde{\Sigma}:\left\{\begin{array}{l}
\dot{q}=\frac{\partial H}{\partial \tilde{p}}\left(\tilde{q}, \tilde{p}, \xi_{i}(i \in \mathbf{m})\right), \\
\dot{\tilde{p}}=-\frac{\partial H}{\partial \tilde{q}}\left(\tilde{q}, \tilde{p}, \xi_{i}(i \in \mathbf{m})\right),
\end{array}\right. \\
& y_{j}=\left[\begin{array}{llll}
1 & 0 & \cdots & 0
\end{array}\right] \xi_{j} \quad(j \in \mathbf{m}),
\end{aligned}
$$

with $A_{i}$ and $B_{i}$ as in Section 3.

Since the linear subsystems $\Sigma_{i}$ are controllable, they can be made asymptotically stable by applying a suitable feedback $v_{i}=F_{i} \xi_{i}$ (i.e., the system can be decoupled by means of a feedback from the class $\mathscr{L}$ such that the linear subsystems of the decoupled system are asymptotically stable; see Section 3 ). If $N^{*}=\{0\}, \widetilde{\Sigma}$ is empty and we arrive at an asymptotically stable system by applying this feedback. (For simple Hamiltonian systems, $N^{*}=\{0\}$ if and only if the number of inputs is equal to the number of configuration coordinates.) The question arises under which conditions this feedback stabilizes the total system (4.3) if $N^{*} \neq\{0\}$. For this purpose we consider the system we obtain from $\tilde{\Sigma}$ by putting $\xi_{i}=0(i \in \mathbf{m})$ :

$$
\left\{\begin{array}{l}
\dot{\tilde{q}}=\frac{\partial \bar{H}}{\partial \tilde{p}}(\tilde{q}, \tilde{p}), \\
\dot{\tilde{p}}=-\frac{\partial \bar{H}}{\partial \tilde{q}}(\tilde{q}, \tilde{p}),
\end{array}\right.
$$

where $\bar{H}(\tilde{q}, \tilde{p})=H(\tilde{q}, \tilde{p}, 0)$.

Assume that $(\tilde{q}, \tilde{p})=(0,0)$ is an equilibrium point of the system (4.3) or, equivalently, that $(\tilde{q}, \tilde{p}, \xi)=(0,0,0)$ is an equilibrium point of $(4.2)$.

Proposition 4.1. Consider the system (4.2). Then:

(i) The equilibrium point $(\tilde{q}, \tilde{p}, \xi)=(0,0,0)$ cannot be made asymptotically stable by applying feedback of the form $v_{i}=F_{i} \xi_{i}$.

(ii) The equilibrium point $(\tilde{q}, \tilde{p}, \xi)=(0,0,0)$ can be made stable by a feedback of the form $v_{i}=F_{i} \xi_{i}$ if and only if $(\tilde{q}, \tilde{p})=(0,0)$ is a stable equilibrium point of (4.3).

In the proof of Proposition (4.1) we use the following result from center manifold theory (see $[\mathrm{C}]$ ).

Proposition 4.2. Consider the system

$$
\left\{\begin{array}{l}
\dot{x}=A x+f(x, z) \\
\dot{z}=B z+g(x, z)
\end{array}\right.
$$

where $x \in \mathbb{R}^{m}, z \in \mathbb{R}^{n}$, and $A$ and $B$ are constant matrices such that all the eigenvalues of $A$ have negative real parts while all the eigenvalues of $B$ have zero real parts. The 
functions $f$ and $g$ are $C^{2}$ with $f(0,0)=D f(0,0)=0$ and $g(0,0)=D g(0,0)=0$ (here $D f$ is the Jacobian matrix of $f$ ). Then:

(i) There exists ancenter manifold for (4.4),

$$
x=h(z), \quad|x|<\delta, \quad \text { where } h \text { is } C^{2} .
$$

The flow on the center manifold is governed by the n-dimensional system

$$
\dot{\eta}=B \eta+g(h(\eta), \eta)
$$

(ii) Assume that the equilibrium point of (4.5) is stable (asymptotically stable) (unstable). Then the equilibrium point of (4.4) is stable (asymptotically stable) (unstable).

Proof of Proposition 4.1. (i) The dynamics of (4.3) are governed by a Hamiltonian vector field and hence, by Liouville's theorem (see $[\mathrm{AM}]),(\tilde{q}, \tilde{p})=(0,0)$ can only be a stable equilibrium point of (4.3) and not an asymptotically stable one. Hence $(\tilde{q}, \tilde{p}, \xi)=(0,0,0)$ cannot be made asymptotically stable.

(ii) (Necessity) By the proof of (i) we see that $(\tilde{q}, \tilde{p}, \xi)=(0,0,0)$ can at most be made a stable equilibrium point of (4.2) and that a necessary condition for stability is that $(\tilde{q}, \tilde{p})=(0,0)$ is a stable equilibrium point of $(4.3)$.

(Sufficiency) Assume that $(\tilde{q}, \tilde{p})=(0,0)$ is a stable equilibrium point of (4.3). Now apply a stabilizing feedback to the linear part of (4.2). After this, feedback (4.2) is of the form (after simplification of the notation)

$$
\begin{aligned}
& \dot{\xi}=A \xi, \\
& \dot{q}=\frac{\partial H}{\partial p}(q, p, \xi), \\
& \dot{p}=-\frac{\partial H}{\partial q}(q, p, \xi),
\end{aligned}
$$

where $A$ is an asymptotically stable matrix.

Taking Taylor series around $(q, p, \xi)=(0,0,0)$ yields a system of the form

$$
\begin{gathered}
\dot{\xi}=A \xi, \\
{\left[\begin{array}{l}
\dot{q} \\
\dot{p}
\end{array}\right]=K\left[\begin{array}{l}
q \\
p
\end{array}\right]+L \xi+g(q, p, \xi),}
\end{gathered}
$$

where $g(0,0,0)=D g(0,0,0)=0$. Moreover, the matrix $K$ has all its eigenvalues on the imaginary axis: $K$ is the state matrix of a linear Hamiltonian system (in fact, it is the state matrix of the linearization of (4.3)), and it is well known (see [AM]) that if a Hamiltonian matrix has an eigenvalue $\lambda \in \mathbb{C}$, then it also has an eigenvalue $-\lambda$. Hence, existence of an eigenvalue of $K$ in the open left half plane would contradict the stability of (4.3).

Since $A$ and $K$ have no common eigenvalues, we can block-diagonalize the linear part of (4.7) by a transformation of the $\xi$-coordinates, thus arriving at a system of the form (4.4). In this case the center manifold is given by $\xi=0$ and hence the flow 
on the center manifold is governed by the system

$$
\left[\begin{array}{l}
\dot{q} \\
\dot{p}
\end{array}\right]=K\left[\begin{array}{l}
q \\
p
\end{array}\right]+g(q, p, 0) .
$$

Noting that (4.8) is equivalent to (4.3), we conclude, from the stability of the equilibrium point $(\tilde{q}, \tilde{p})=(0,0)$ of $(4.3)$ and Proposition 4.2 , that $(\tilde{q}, \tilde{p}, \xi)=(0,0,0)$ can be made a stable equilibrium point of (4.2).

Using Proposition 4.1 we can give the following sufficient condition for stabilizabilty of (4.2):

Theorem 4.1. Consider system (4.2). Let the function $\bar{H}(\tilde{q}, \tilde{p})$ be defined by $\bar{H}(\tilde{q}, \tilde{p}):=$ $H(\tilde{q}, \tilde{p}, 0)$. Then $(\tilde{q}, \tilde{p}, \xi)=(0,0,0)$ can be made a stable (not asymptotically stable) equilibrium point of $(4.2)$ if $\bar{H}$ has an isolated local minimum $(\tilde{q}, \tilde{p})=(0,0)$.

Proof. It is straightforward to check that $\dot{H}=0$. Hence, if $\bar{H}$ has an isolated local minimum at $(\tilde{q}, \tilde{p})=(0,0)$, then $\bar{H}$ is a Lyapunov function, which implies that $(\tilde{q}, \tilde{p})=(0,0)$ is a stable equilibrium point of $(4.3)$. Hence, by Proposition $4.1,(\tilde{q}, \tilde{p}, \xi)$ can be made a stable equilibrium point of (4.2).

For the stability of a Hamiltonian system it is not necessary that the Hamiltonian has an isolated local minimum at the stable equilibrium point (see $[\mathrm{AM}]$ ), so the converse of Theorem 4.1 is not true in general. However, for simple Hamiltonian systems (see Definition 2.1) we can make a statement about the converse of Theorem 4.1 .

Consider a simple Hamiltonian system with $m$ imputs (see Definition 2.1). As remarked before, it follows from $\operatorname{rank} A=m$ that the distribution $G=$ $\operatorname{span}\left(X_{C_{1}}, \ldots, X_{C_{m}}\right)$ has constant dimension $m$. Therefore the codistribution $\operatorname{span}\left\{d C_{1}, \ldots, d C_{m}\right)$ also has constant dimension $m$, and hence we can find canonical coordinates $(q, p)$ such that $C_{i}(q)=q_{i}, i \in \mathbf{m}$.

Because for a simple Hamiltonian system $\rho_{i}=1, i \in \mathbf{m}$, we then have $\xi=\left(q_{1}, \dot{q}_{1}, \ldots, q_{m}, \dot{q}_{m}\right)$. Moreover, we can take $\tilde{q}=\left(q_{m+1}, \ldots, q_{n}\right)^{\mathrm{T}}$ and $\tilde{p}=$ $\left(p_{m+1}, \ldots, p_{n}\right)^{\mathrm{T}}$. Denoting $\bar{q}=\left(q_{1}, \ldots, q_{m}\right)^{\mathrm{T}}$ and

$$
G^{-1}(q)=\left[\begin{array}{ll}
G_{11}(q) & G_{12}(q) \\
G_{12}^{\mathrm{T}}(q) & G_{22}(q)
\end{array}\right],
$$

where $G_{11}(q)$ is an $m \times m$ matrix (in fact, $-G_{11}$ equals the decoupling matrix $A$ ) and $G_{22}(q)$ is an $n-m \times n-m$ matrix, we get

$$
H(\tilde{q}, \tilde{p}, \xi)=H(\tilde{q}, \tilde{p}, \bar{q}, \dot{\bar{q}})=\frac{1}{2} \dot{\bar{q}}^{\mathrm{T}} G_{1}(q) \dot{\bar{q}}+\frac{1}{2} \tilde{p}^{\mathrm{T}} G_{2}(q) \tilde{p}+V(\bar{q}, \tilde{q}),
$$

where $G_{1}(q)=G_{11}^{-1}(q)$ and $G_{2}(q)=G_{22}(q)-G_{12}^{\mathrm{T}}(q) G_{11}^{-1}(q) G_{12}(q)$. Hence, without having to calculate $N^{*}$ explicitly we obtain

$$
\bar{H}(\tilde{q}, \tilde{p})=H(\tilde{q}, \tilde{p}, 0)=\frac{1}{2} \tilde{p}^{\mathrm{T}} G_{2}(q) \tilde{p}+V(0, \tilde{q}) .
$$

It is easy to check that $G_{2}(q)$ is again positive definite for all $q$. 
Proposition 4.3. Consider a Hamiltonian function $H$ belonging to a simple Hamiltonian system (see Definition 2.1). Assume that the origin is an equilibrium point of $\dot{x}=X_{H}(x)$. Then for all potential energy functions $V(q)$ in an open and dense subset of $C^{\infty}(Q)$ (with the $C^{+\infty}$-Whitney topology), if the origin is a stable equilibrium, $H$ has an isolated minimum at the origin.

Proof. Consider a Hamiltonian belonging to a simple Hamiltonian system: $H(q, p)=\frac{1}{2} p^{\mathrm{T}} G^{-1}(q) p+V(q)$, where $G^{-1}(q)$ is positive-definite for all $q$. Assume that the origin is an equilibrium point of $\dot{x}=X_{H}(x)$, i.e., $d V(0)=0$. The Hessian $H^{*}$ of $H$ in $(q, p)=(0,0)$ is

$$
H^{*}=\left[\begin{array}{cc}
W & 0 \\
0 & P
\end{array}\right] \text { where } W=\frac{\partial^{2} V}{\partial q^{2}}(0,0) \text { and } P=G(0) .
$$

Furthermore, stability of $(q, p)=(0,0)$ also means that $(\hat{q}, \hat{p})=(0,0)$ is a stable equilibrium point of the linearization of $\dot{x}=X_{H}(x)\left(x=(q, p)^{\mathrm{T}}\right)$ around $(q, p)=$ $(0,0)$. This linearized system is

$$
\left[\begin{array}{c}
\dot{q} \\
\dot{\hat{p}}
\end{array}\right]=\left[\begin{array}{cc}
0 & P \\
-W & 0
\end{array}\right]\left[\begin{array}{l}
\hat{q} \\
\hat{p}
\end{array}\right] .
$$

Now, because of the fact that $P$ is positive-definite, stability of (4.10) implies that, for $V(q)$ in an open and dense subset of $C^{\infty}(Q)$, the matrix $W$ is positive-definite. Hence, for all these $V(q), H^{*}$ is positive definite and therefore $H$ has an isolated local minimum at $(q, p)=(0,0)$.

Combining the results above gives our main theorem.

Theorem 4.2. Consider the Hamiltonian system (4.1). Assume that the input-output decoupling problem is solvable and that the distribution spanned by the input vector fields is involutive. Let $\bar{H}$ be defined as the Hamiltonian $H$ restricted to $N^{*}$. Assume that the origin is an equilibrium of $\dot{\bar{x}}=X_{\bar{H}}(\bar{x})$. Finally, let coordinates $(\tilde{q}, \tilde{p}, \xi)$ for $M$ be given as in Section 3. Then:

(i) Application of a decoupling feedback from the class $\mathscr{L}$ cannot result in an asymptotically stable equilibrium point $(\tilde{q}, \tilde{p}, \tilde{\xi})=(0,0,0)$.

(ii) There is a decoupling feedback from the class $\mathscr{L}$ which renders $(\tilde{q}, \tilde{p}, \xi)=$ $(0,0,0)$ a stable equilibrium point if and only if $\dot{\bar{x}}=X_{\bar{H}}(\bar{x})$ has a stable equilibrium at the origin.

(iii) If $\bar{H}$ has an isolated minimum at the origin, then $\dot{\bar{x}}=X_{\bar{H}}(\bar{x})$ has a stable equilibrium at the origin.

(iv) If, moreover, (4.1) is a simple Hamiltonian system, then, for all $V(q)$ in an open and dense subset of $C^{\infty}(Q)$, the existence of a decoupling feedback from the class $\mathscr{L}$ that stabilizes $(\tilde{q}, \tilde{p}, x)=(0,0,0)$ implies that $\bar{H}$ has an isolated minimum at the origin.

Remark. The theorem can be easily extended to the following generalized version of system equations (4.1). Assume that the input vector fields in (4.1) are not 
necessarily given by $X_{c_{j}}$, but instead take the more general form

$$
X_{P_{j}\left(C_{1}, \ldots, c_{m}\right)} \quad(j \in \mathbf{m}),
$$

where the mapping $P=\left(P_{1}, \ldots, P_{m}\right): \mathbb{R}^{m} \rightarrow \mathbb{R}^{m}$ is assumed to be a diffeomorphism. (For example, this may happen in the case of robot manipulators. Generally, the input torques correspond to the joint coordinates, but the outputs may be given in task space coordinates. In the case of an equal number of inputs and outputs the joint coordinates are usually related to the task coordinates by a transformation which is invertible except for some singular points.) Then the characteristic numbers $\rho_{i}$ are the same as if the input vector fields would equal $X_{c_{j}}, j \in \mathbf{m}$. Moreover, the decoupling matrix in this generalized case is given as

$$
\left(\begin{array}{ccc}
\left\{C_{1}, \mathrm{ad}_{H}^{\rho_{1}} C_{1}\right\} & \cdots & \left\{C_{m}, \operatorname{ad}_{H}^{\rho_{1}} C_{1}\right\} \\
\vdots & & \vdots \\
\left\{C_{1}, \operatorname{ad}_{H}^{\rho_{m}} C_{m}\right\} & \cdots & \left\{C_{m}, \operatorname{ad}_{H}^{\rho_{m}} C_{m}\right\}
\end{array}\right)\left(\begin{array}{ccc}
\frac{\partial P_{1}}{\partial y_{1}} & \cdots & \frac{\partial P_{m}}{\partial y_{1}} \\
\vdots & & \vdots \\
\frac{\partial P_{1}}{\partial y_{m}} & \cdots & \frac{\partial P_{m}}{\partial y_{m}}
\end{array}\right)
$$

and hence, since $P$ is a diffeomorphism, the decoupling matrix has full rank if and only if the decoupling matrix for the system with input vector fields $X_{C_{j}}$ has full rank. A decoupling feedback from the class $\mathscr{L}$ brings the system into the same form (4.2), and so Theorem 4.2 also applies to this more general case.

Example 4.1. Consider a simple robot arm, consisting of two unit masses attached to massless links of length one, where the first link is flexible. In order to model this flexibility we divide the first link in two auxiliary links, one of length $\varepsilon$ and the other of length $1-\varepsilon$, connected by a torsional spring with spring constant $k$ (Fig. 2). We apply a torque $u_{1}$ on the auxiliary link, hence in the Hamiltonian formulation the

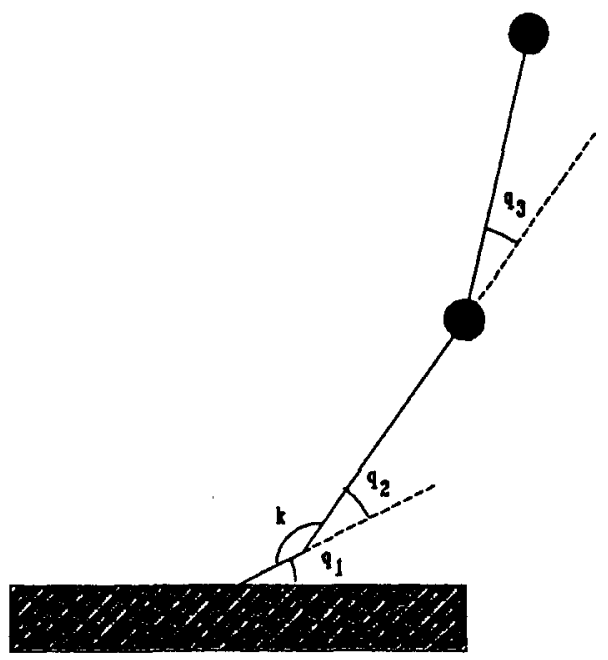

Fig. 2. Robot arm model. 
first input vector field will be $X_{D_{1}}$, where $D_{1}(q)=q_{1}$. On the second link we apply a torque $u_{2}$. Because of the flexibility of the first link, we assume that the motor exerting this torque is situated in the base of the robot arm and that the torque is transfered by means of a chain belt. Since this introduces reaction forces (see [AS]), in the Hamilton formulation the second input vector field will be $X_{D_{2}}$, where $D_{2}(q)=q_{1}+q_{3}$. To guarantee positive definiteness of the kinetic energy matrix we have to introduce an auxiliary mass $\delta(\ll 1)$ located at the joint between the first and second auxiliary link.

We are now interested in the stability properties of the (unstable) equilibrium point $\left(q_{1}, q_{2}, q_{3}\right)=(\pi / 2,0,0)$ after we have applied a decoupling feedback from the class $\mathscr{L}$. This means that we are interested in the outputs $C_{1}(q)=q_{1}-\pi / 2$, $C_{2}(g)=q_{3}$ rather than $D_{1}$ and $D_{2}$. However, there exists a diffeomorphism $P$ : $\mathbb{B}^{2} \rightarrow \mathbb{R}^{2}$ such that $P\left(C_{1}, C_{2}\right)=\left(D_{1}, D_{2}\right)$. Hence, by the remark above, the characteristic numbers and the class $\mathscr{L}$ will be the same with both choices of outputs.

Calculation (using a program such as REDUCE) yields

$$
\begin{gathered}
N^{*}=\left\{(q, p) \mid q_{1}=\frac{\pi}{2}, q_{3}=0,\right. \\
\left.p_{1}=\left(\frac{(3-2 \varepsilon) \varepsilon \cos q_{2}}{2 \varepsilon^{2}-6 \varepsilon+5}+1\right) \cdot p_{2}, p_{3}=\frac{2-\varepsilon}{2 \varepsilon^{2}-6 \varepsilon+5} \cdot p_{2}\right\}, \\
\bar{H}\left(q_{2}, p_{2}\right)=\frac{1}{2\left(2 \varepsilon^{2}+6 \varepsilon+5\right)} \cdot p_{2}^{2}+2 \varepsilon g+(3-2 \varepsilon) g \cos q_{2}+\frac{1}{2} \cdot k q_{2}^{2}
\end{gathered}
$$

(where we have put $\delta=0)$. It is obvious that $\left(q_{2}, p_{2}\right)=(0,0)$ is an equilibrium point of $\dot{x}=X_{\bar{H}}(x)$. The Hessian of $\bar{H}$ in this equilibrium point is

$$
\left[\begin{array}{cc}
k-(3-2 \varepsilon) g & 0 \\
0 & \frac{1}{2 \varepsilon^{2}+6 \varepsilon+5}
\end{array}\right] \text {. }
$$

Hence $\bar{H}$ has an isolated minimum at $\left(q_{2}, p_{2}\right)=(0,0)$ if $k>(3-2 \varepsilon) g$, i.e., if the flexibility is not too big. Thus $\left(q_{2}, p_{2}\right)=(0,0)$ is a stable equilibrium point of the dynamics governed by $X_{\bar{H}}$. (It is easy to check (e.g., by using Lyapunov's first method) that $\left(q_{2}, p_{2}\right)=(0,0)$ is an unstable equilibrium point if $k \leq(3-2 \varepsilon) g$.) Summarizing, $\left(q_{1}, q_{2}, q_{3}\right)=(\pi / 2,0,0)$ can be made a stable equilibrium point by application of a decoupling feedback from the class $\mathscr{L}$ if and only if $k>(3-2 \varepsilon) g$.

\section{Conclusions}

We have derived a new "canonical form" for input-output decoupled systems for which the distribution spanned by the input vector fields is involutive. We have shown that for Hamiltonian systems, by using a decoupling feedback from the easily computable class $\mathscr{L}$, the stability properties of the resulting system can be investigated in an explicit and straightforward way. For simple Hamiltonian systems, which are most often encountered in practice, the procedure is very easy. In many nonlinear examples we have dealt with (e.g., robot systems) the stability properties 
cannot be improved by looking for decoupling feedbacks outside class $\mathscr{L}$. However, the study of fixed dynamics and the computability of a decoupling feedback outside class $\mathscr{L}$ appears promising in the Hamiltonian case and remains for further research. Finally, we note that the stability notion dealt with here is fully local, although the decoupling property and the normal form (3.10) are global. In general, the problems concerning a more global version of stability in this context seem to be delicate, see [S4]; however, in the present case a Lyapunov approach based on the Hamiltonian $H(q, p, \xi)$ could be of help (see $[\mathrm{T}]$ ).

\section{References}

[AM] R. Abraham and J. E. Marsden, Foundations of Mechanics, 2nd edn., Benjamin/Cummings, Reading, MA, 1978.

[A] D. Aeyels, Stabilization of a class of nonlinear systems by a smooth feedback control, Systems Control Lett., 5 (1985), 289-294.

[AS] H. Asada and J.-J. E. Slotine, Robot Analysis and Control, Wiley, New York, 1986.

[B] A. Bacciotti, The local stabilizability problem for nonlinear systems, IMA J. Math. Control Inform., 5 (1988), 27-39.

[BB] A. Bacciotti and P. Boieri, Linear stabilizability of planar nonlinear systems, Math. Control Signals Systems (to appear).

[BI1] C. I. Byrnes and A. Isidori, Local stabilization of minimum phase nonlinear systems, Systems Control Lett., 11 (1988), 9-17.

[BI2] C. I. Byrnes and A. Isidori, New results and examples in nonlinear feedback stabilization, Systems Control Lett. 12 (1989), 437-442.

[C] J. Carr, Applications of Centre Manifold Theory, Springer-Verlag, New York, 1981.

[CS] P. E. Crouch and A. J. van der Schaft, Variational and Hamiltonian Control Systems, Lecture Notes in Control and Information Sciences, Vol. 101, Springer-Verlag, Berlin, 1987.

[G] E. G. Gilbert, The decoupling of multivariable systems by state variable feedback, SIAM J. Control Optim., 7 (1969), 50-64.

[HG] I. J. Ha and E. G. Gilbert, A complete characterization of decoupling control laws for a general class of nonlinear systems, IEEE Trans. Automat. Control, 31 (1986), 823-830.

[I] A. Isidori, Nonlinear Control Systems: An Introduction, Lecture Notes in Control and Information Sciences, Vol. 72, Springer-Verlag, Berlin, 1985.

[IG] A. Isidori and J. W. Grizzle, Fixed modes and nonlinear noninteracting control with stability, IEEE Trans. Automat. Control, 33 (1988), 907-914.

[M1] R. Marino, High-gain feedback in nonlinear control systems, Internat. J. Control, 42 (1985), 1369-1385.

[M2] R. Marino, Feedback stabilization of single-input nonlinear systems, Systems Control Lett., 10 (1988), 201-206.

[MBE] R. Marino, W. M. Boothby, D. L. Elliott, Geometric properties of linearizable controi systems, Math. Systems Theory, 18 (1985), 97-123.

[NS] H. Nijmeijer and J. M. Schumacher, The regular local noninteracting control problem for nonlinear control systems, SIAM J. Control Optim., 24 (1986), 1232-1245.

[S1] A. J. van der Schaft, System Theoretic Descriptions of Physical Systems, CWI Tract 3, CWI, Amsterdam, 1984.

[S2] A. J. van der Schaft, On feedback control of Hamiltonian systems, in Theory and Applications of Nonlinear Control Systems (C. Byrnes and A. Lindquist, eds), pp. 273-290, Elsevier, Amsterdam, 1986.

[S3] A. J. van der Schaft, Equations of motion for Hamiltonian systems with constraints, J. Phys. A, 20 (1987), 3271-3277.

[S4] H. J. Sussmann, Some examples on the stabilizability of globally minipum phase systems, IEEE Trans. Automat. Control (to appear).

[T] J. Tsinias, Sufficient Lyapunov-like conditions for stabilization, Math. Control Signals Systems, 2 (1989), 343-357. 